\title{
Monitoreo continuo de glucosa: indicaciones, interpretación de datos y toma de decisiones terapéuticas Recomendaciones de expertos
}

\author{
Continuous glucose monitoring: indications, \\ data interpretation and making therapeutic decisions \\ Expert recommendations
}

\author{
León E. Litwak ${ }^{1}$, Nancy Carreño², Rodrigo Carnero ${ }^{3}$, Alejandro Dain ${ }^{4}$, Luis A. Grosembacher ${ }^{5}$, \\ Carla Musso $^{6}$, Adrián Proietti ${ }^{7}$, Lucas Rista ${ }^{8}$, Gabriela Rovira ${ }^{9}$, Graciela Rubin ${ }^{10}$, María L. Ruiz ${ }^{11}$, \\ Antonio Saleme ${ }^{12}$, Liliana Trifone ${ }^{13}$, José E. Costa Gil ${ }^{14}$
}

\begin{abstract}
RESUMEN
Las herramientas para evaluar el grado de control glucémico se modificaron últimamente. La hemoglobina glicosilada ( $\mathrm{HbA} 1 \mathrm{c})$, parámetro de referencia (gold standard), refleja el control glucémico de los últimos tres meses de manera retrospectiva, sin expresar la variabilidad glucémica. El automonitoreo glucémico capilar (AGC) brinda información inmediata y prospectiva, pero dispone de pocos datos glucémicos para generar promedios y desviaciones estándares representativas. No detecta tendencias y tiene limitaciones para obtener datos nocturnos o durante la actividad física. Es invasivo y muchas veces rechazado. Contrariamente, el monitoreo continuo de glucosa (MCG) mide la glucosa instantáneamente, y muestra sus tendencias y su variabilidad en forma continua, incorporando nuevas métricas de control.

Mediante el perfil ambulatorio de glucosa (PAG) se analizan los patrones del control glucémico durante el sueño, los ayunos prolongados, la actividad física y las intercurrencias, expresándolos como curvas con sus desviaciones estándar durante períodos de horas (8 a 24 horas) o días (7, 14, 30 y 90 días). El PAG contiene las siguientes métricas: porcentaje de tiempo en rango TIR (del inglés, time in range), porcentaje de tiempo por encima del rango TAR (del inglés, time above range), porcentaje de tiempo por debajo del rango o hipoglucemia TBR (del inglés, time below range) y coeficiente de variabilidad (\%CV). La información continua permite tomar decisiones inmediatas, ya sea con la ingesta de carbohidratos o con la aplicación de insulina. EI MCG con terapéuticas insulínicas inyectables (TII) o bomba portable de insulina (BPI) es una herramienta muy útil y complementaria para el tratamiento de la diabetes mellitus tipo 1 (DM1) y la DM2 en la insulinoterapia. Su utilización se asoció con descensos significativos en la HbA1c, disminución de la variabilidad glucémica, reducción de las hipoglucemias totales y nocturnas, y mejoría de la calidad de vida en estos pacientes. Nuestro propósito como grupo de expertos es generar una guía práctica para regular la implementación del MCG.
\end{abstract}

Palabras clave: diabetes; monitoreo continuo de glucosa; control glucémico; insulinoterapia intensificada.

Revista de la Sociedad Argentina de Diabetes 2020; Vol. 54 (140-154)

\section{ABSTRACT}

The tools to assess the degree of glycemic control were modified lately. Glycosylated hemoglobin (HbA1C), the gold standard, reflects the glycemic control of the last 3 months retrospectively, without expressing glycemic variability. Selfblood glucose monitoring (SBGM) provides immediate and prospective information, but has little glycemic data to generate representative averages and standard deviations. It does not detect trends and has limitations to obtain nocturnal data or during physical activity. It is invasive and often rejected. On the contrary, continuous glucose monitoring (CGM), allows to measure glucose instantly, shows your trends and variability continuously, incorporating new control metrics. The ambulatory glucose profile (AGP) analyzes the patterns of glycemic control during sleep, prolonged fasting, physical activity and intercurrences, expressing them as curves with their standard deviations during periods of hours (8 to 24 hours) or days (7, 14, 30 and 90 days). The AGP contains the following metrics: percentage time in range (TIR), percentage time above range $\mathrm{mg} / \mathrm{dl}$ (TAR), percentage time below range or hypoglycemia (TBR) and coefficient of variation (\% CV). CGM with IIT or continuous subcutaneous insulin infusion (CSII), is a very useful and complementary tool for the treatment of DM1 and DM2 in insulin therapy. Its use was associated with significant decreases in $\mathrm{HbA1C}$, decreased glycemic variability, reduction of total and nocturnal hypoglycemia and improvement of the quality of life in these patients. Our aim as a group of experts is to generate a practical guide to regulate the implementation of the CGM.

Key words: diabetes; continuous glucose monitoring; glycemic control; intensive insulin therapy.

Revista de la Sociedad Argentina de Diabetes 2020; Vol. 54 (140-154) 
1 Médico Endocrinólogo, Servicio de Endocrinología, Metabolismo y Medicina Nuclear, Hospital Italiano de Buenos Aires, Ciudad Autónoma de Buenos Aires, Argentina

2 Médica especialista en Clínica Médica, Nutrición y Diabetes, Fundación Argentina Diabetes, Secretaria del Comité de Innovación en Diabetes, Sociedad Argentina de Diabetes (SAD), Ciudad Autónoma de Buenos Aires, Argentina

3 Médico especialista en Medicina General, Máster en Endocrinología Clínica, Jefe del Servicio de Endocrinología y Diabetes, Instituto Médico de Río Cuarto, Comité de Innovación en Diabetes, Sociedad Argentina de Diabetes (SAD) Córdoba, Argentina

4 Doctor en Medicina, Cirugía, especialista en Medicina Interna, Nutrición Clínica y Diabetología, Profesor Universitario, Profesor adjunto Cátedra de Histología, Universidad Nacional Villa María y Docente de la Universidad Nacional de Córdoba (UNC), coordinador de la Maestría en Diabetes y Nutrición (UNC, Área Nutrición), staff Clínica Universitaria Reina Fabiola de Córdoba, Córdoba, Argentina

5 Médico Endocrinólogo, Jefe de la Sección Diabetes, Servicio de Endocrinología, Metabolismo y Medicina Nuclear, Hospital Italiano de Buenos Aires, Ciudad Autónoma de Buenos Aires, Argentina

6 Médica Endocrinóloga, Coordinadora del Área Diabetes, Fundación Favaloro, Ciudad Autónoma de Buenos Aires, Argentina

7 Especialista en Medicina Interna, Médico Endocrinólogo, Director Médico del Instituto Integral de Diabetes y Tecnología Aplicada (IDTA), Director del Curso de Tecnología aplicada a la Diabetes, Sociedad Argentina de Diabetes (SAD), Ciudad Autónoma de Buenos Aires, Argentina

8 Magister en Diabetes, miembro del Comité de Innovación y Presidente Capítulo Litoral, Sociedad Argentina de Diabetes (SAD), Centro de Diabetes y Nutrición de Rosario, Presidente Fundación Paul Langerhans, Santa Fe, Argentina

9 Médica Endocrinóloga, Coordinadora del Área Tecnología y Diabetes, Servicio de Endocrinología, Metabolismo, Nutrición y Diabetes, Hospital Británico de Buenos Aires, Ciudad Autónoma de Buenos Aires, Argentina

10 Médica Internista, experta en Diabetes, Servicio de Diabetología y Nutrición, Hospital Privado Universitario de Córdoba, Coordinadora del Comité de Innovación en Diabetes, Sociedad Argentina de Diabetes (SAD), Córdoba, Argentina
11 Médica de Familia especializada en Diabetes, Centro Consultores en Diabetes y Enfermedades Metabólicas (CODIME), Coordinadora del Curso de Tecnología Aplicada a la Diabetes, Sociedad Argentina de Diabetes (SAD), Ciudad Autónoma de Buenos Aires, Argentina

12 Magister en Diabetes, Posgrado Facultad de Ciencias Médicas, Universidad Favaloro, Médico especialista en Clínica Médica, miembro del Comité de Innovación y Diabetes, Sociedad Argentina de Diabetes (SAD), Jefe del Servicio de Endocrinología y Metabolismo, Sanatorio Santa Fe, Santa Fe, Argentina

13 Médica Pediatra especialista en Nutrición, Jefa de la Sección Nutrición y Diabetes, Hospital de Niños Ricardo Gutiérrez, Ciudad Autónoma de Buenos Aires, Argentina

14 Doctor en Medicina, Posgrado Facultad de Ciencias Médicas, Universidad Favaloro, Ciudad Autónoma de Buenos Aires, Argentina

Contacto del autor: León E. Litwak

E-mail: leon.litwak@hospitalitaliano.org.ar

Correspondencia: Lafinur 3050 piso 8 depto. 2 (C1425FAD), Ciudad Autónoma de Buenos Aires, Argentina

Fecha de trabajo recibido: 26/06/20

Fecha de trabajo aceptado: 10/08/20

Conflictos de interés: el Dr. León E. Litwak es miembro del consejo asesor y disertante de Laboratorios Abbott. El Dr. Rodrigo Carnero es disertante de Medtronic. El Dr. Alejandro Dain es disertante de Laboratorios Roche y Abbott. El Dr. Luis A. Grosembacher es disertante de Laboratorios Abbott y Medtronic. La Dra. Carla Musso es disertante de Laboratorios Roche y Abbott. El Dr. Adrián Proietti es disertante de Laboratorios Abbott y Medtronic. El Dr. Lucas Rista es disertante de Laboratorios Abbott y Roche. La Dra. Gabriela Rovira es disertante de Laboratorios Abbott y Roche. La Dra. Graciela Rubin es asesora médica de Medtronic. La Dra. María L. Ruiz es disertante de Laboratorios Roche y Abbott. El Dr. Antonio Saleme es disertante de Laboratorios Abbott. La Dra. Liliana Trifone es disertante de Medtronic. El Dr. José E. Costa Gil es miembro del Consejo asesor y disertante de Laboratorios Abbott. La Dra. Nancy Carreño no tiene conflictos de interés.

\section{INTRODUCCIÓN}

Los criterios para considerar el adecuado control de la glucemia en pacientes con diabetes mellitus (DM) se han modificado sustancialmente en los últimos años, tanto en cuanto a las metas (que no son iguales para todas las guías de sociedades científicas) como en cuanto a los recursos que se utilizan para evaluar el nivel de control.

La hemoglobina glicosilada (HbA1c), considerada aún como el parámetro de referencia (gold standard, si bien refleja el control glucémico de los últimos tres meses, lo hace en forma retrospectiva, sin expresar la variabilidad glucémica ni sus tendencias. Su determinación puede estar afectada por hemoglobinopatías, condiciones que alteran la eritropoyesis, así como por la técnica de laboratorio empleada para su determinación.
Esto podría inducir a tomar decisiones terapéuticas inadecuadas y en forma tardía'. Por esta razón, los valores de $\mathrm{HbA} 1 \mathrm{c}$ deben analizarse en conjunto con otras métricas de importancia para lograr un correcto análisis. Por otra parte, el automonitoreo glucémico capilar (AGC), que representó un gran avance para el control de la DM y aún es una herramienta muy útil, si bien otorga información inmediata y prospectiva, sólo permite disponer de pocos datos glucémicos diarios (promedio 3 a 5 diarios, con un máximo de 8 a 9 en embarazadas con DM), con sus respectivos promedios y desviaciones estándar, pero no informa acerca de las tendencias (son sólo valores estáticos). Presenta, además, limitaciones para obtener datos nocturnos o durante la actividad física, y aún es un método invasivo y no pocas veces rechazado por los 
pacientes². Sin embargo, los últimos avances permitieron mejorar su precisión y exactitud. La Sociedad Argentina de Diabetes (SAD) emitió un documento de Opiniones y Recomendaciones que sigue vigente para la implementación del $\mathrm{AGC}^{3,4}$.

En la actualidad, el monitoreo continuo de glucosa (MCG), con sus diferentes tecnologías disponibles, permite conocer no sólo el valor de glucosa en forma instantánea, sino también sus tendencias mediante flechas que ayudan a visualizarlas en la pantalla del lector. Facilita, también, disponer de los niveles de glucosa históricos, incluida su variabilidad. Además puede almacenar datos puntuales importantes, como el horario de las ingestas y su contenido, las dosis y los horarios de aplicación de insulina, el tipo de ejercicio, entre otros ${ }^{4}$. El MCG ha incorporado, para estimar el nivel de control glucémico, nuevas métricas (ver más adelante la descripción más completa) tales como el porcentaje de tiempo en rango (TIR, del inglés, time in range), el tiempo por encima del rango (>180 mg/dl; TAR, del inglés, time above range) y el tiempo por debajo del rango (hipoglucemia: $<70 \mathrm{mg} / \mathrm{dl}$; TBR, del inglés, time below range). Otro importante parámetro informado es la variabilidad de los niveles de glucosa que se expresa como coeficiente de variabilidad (\%CV). Toda esta información se obtiene en forma permanente y continua para tomar decisiones terapéuticas inmediatas y más precisas que con el AGC convencional, ya sea con la ingesta preventiva de carbohidratos o con la realización de correcciones con insulina de acción rápida. Agrega la posibilidad, mediante el análisis de los patrones generales del control glucémico denominado perfil ambulatorio de glucosa (PAG, ver más adelante), de realizar los ajustes particularmente en períodos del día en los que es difícil efectuar mediciones de glucosa (durante el sueño, períodos prolongados de ayuno por saltear comidas, durante la actividad física, etcétera).

La utilización del MCG mejora el control glucémico, disminuye su variabilidad y reduce hipoglucemias graves, sobre todo nocturnas, con mejoría significativa de la calidad de vida. Asimismo, los beneficios clínicos del uso de MCG en personas con DM son independientes de los medios y las estrategias de administración de insulina ${ }^{5}$. Resta evaluar con nuevas investigaciones la factibilidad de reducir significativamente la aparición o la progresión de las principales complicaciones crónicas de la DM, ya que existe fuerte evidencia de asociación entre el TIR y el \%CV con la enfermedad cardiovascular, la vulnerabilidad de placa y las modificaciones epigenéticas que predisponen a la disfunción endotelial ${ }^{5}$. La incorporación del MCG, tanto con sistemas con técnicas de lectura en tiempo real (MCG-RTS, del inglés, real time system) como con técnicas de lectura intermitente (flash) (MCG-IS/Flash, del inglés, intermittently scanned), ha crecido de forma rápida durante los últimos años como resultado de las mejoras técnicas relacionadas con la seguridad, la precisión, la exactitud y la facilidad de uso de dichos sensores.

Dada la accesibilidad, aceptabilidad y facilidad de uso, los diferentes sistemas de MCG comenzaron a incorporarse como parte del tratamiento de los pacientes con DM en insulinoterapia, sin que existan aún indicaciones precisas para su uso y sin un adecuado entrenamiento en la interpretación de los datos proporcionados. Esto se observó tanto por parte de los pacientes como también de los médicos tratantes, lo que produjo errores terapéuticos y la utilización inadecuada de este sistema.

El propósito de este documento es generar una guía práctica basada en la opinión de un grupo de expertos, para colaborar con su apropiada implementación y aprovechar al máximo estos nuevos recursos tecnológicos.

\section{Indicaciones del MGC}

Los sistemas de MCG están constituidos por un sensor que informa de forma ininterrumpida los niveles de glucosa en líquido intersticial y un módulo receptor que permite su lectura y almacenamiento. Los modelos de tiempo real (MCGRTS) permiten observar continuamente los datos, mientras que los sistemas intermitentes (MCG-IS/ Flash) los muestran siempre y cuando se acerque el módulo receptor al sensor, lo que genera una lectura voluntaria e intermitente.

Se recomienda el MCG-RTS (los más difundidos son Dexcom y Medtronic) o el MCG-IS/Flash (FreeStyle Libre). Este último es actualmente el único sistema accesible en Argentina no integrado a un infusor de insulina y se recomienda siempre y cuando los pacientes tengan accesibilidad al sistema, expresen su deseo para incorporarlo, demuestren capacidad para utilizarlo, sean asistidos por un equipo profesional entrenado en su uso y se respeten las indicaciones para ello. 


\section{Indicaciones principales para el uso del MCG}

- Adultos, adolescentes y niños la partir de 4 años para FreeStyle Libre y de 2 años para Dexcom) con DM1 que no logran adecuado control glucémico (objetivo personalizado de $\mathrm{HbA} 1 \mathrm{c})^{6,7}$.

- Adultos, adolescentes y niños (a partir de 4 años para FreeStyle Libre y de 2 años para Dexcom) con DM1 con adecuado control, pero que presentan hipoglucemias leves/moderadas reiteradas o prolongadas o bien nocturnas/graves, sin evidenciar falta de percepción de hipoglucemia (unawareness) $)^{8,9}$

- Niños (a partir de 4 años para FreeStyle Libre y de 2 años para Dexcom) y adolescentes con DM1 bajo tratamiento intensivo, tanto con múltiples dosis de insulina (MDI), mediante bomba portable de infusión continua de glucosa (BPI), o con hipoglucemias frecuentes asintomáticas ${ }^{10}$.

- Adultos, adolescentes y niños (a partir de 4 años para FreeStyle Libre y de 2 años para Dexcom) con DM1 que experimentan gran variabilidad glucémica durante el mismo día o entre diferentes días.

- Niños (a partir de 4 años para FreeStyle Libre y de 2 años para Dexcom) con actividad física intensa y variable ${ }^{10}$.

- Adultos y adolescentes con dificultades para el control de la glucosa durante el ejercicio y el estrés?.

- Pacientes con incapacidad física o psicológica para realizar AGC tradicional en forma continua, aunque deben mantener la capacidad de realizar mediciones de glucemia capilar ante la presencia de síntomas.

- Pacientes con DM2 bajo tratamiento intensificado con insulina e inadecuado control glucémico o con hipoglucemias leves/moderadas reiteradas o nocturnas/graves, sin evidenciar falta de percepción de hipoglucemias (unawareness), o bien que experimenten gran variabilidad glucémica dentro del día o entre diferentes días.
Debe considerarse que las evidencias para recomendar el MCG-RTS o el MCG-IS/Flash en personas con DM2 si bien son, por ahora, escasas, hay recientes estudios que demuestran su efectividad en estos pacientes ${ }^{5,11,12,13}$.

- Embarazadas con DM1, complementando, pero no reemplazando, al AGC, lo que permite sumar las fortalezas de los sistemas y alcanzar mayor control de la glucosa durante el embarazo ${ }^{14,15}$.

\section{Indicaciones relativas del MCG}

- Mujeres con diabetes gestacional (aún hay escasa evidencia).

- Embarazadas con DM2 (aún hay escasa evidencia).

- Niños menores de 2 años o 4 según el sistema de monitoreo.

- Personas con enfermedades psiquiátricas caracterizadas por trastornos obsesivos o bien conductas alimentarias inadecuadas.

- Personas que no puedan o no deseen usarlo en forma continua por razones psicológicas, económicas o estéticas.

\section{Lectura e interpretación de resultados}

Se sugiere dividir en dos partes la lectura e interpretación del MCG: la primera se refiere al PAG, que informa sobre la situación actual del control glucémico del paciente en forma sencilla y esquematizada, mientras que la segunda parte muestra los valores de glucosa en los días previos, cuyo análisis brindará curvas de tendencias y datos más precisos de situaciones particulares para su interpretación y posterior toma de conductas terapéuticas (acción). Es un requisito muy importante que los profesionales que interpreten los resultados y determinen las modificaciones terapéuticas hayan sido entrenados para la utilización del MCG. 


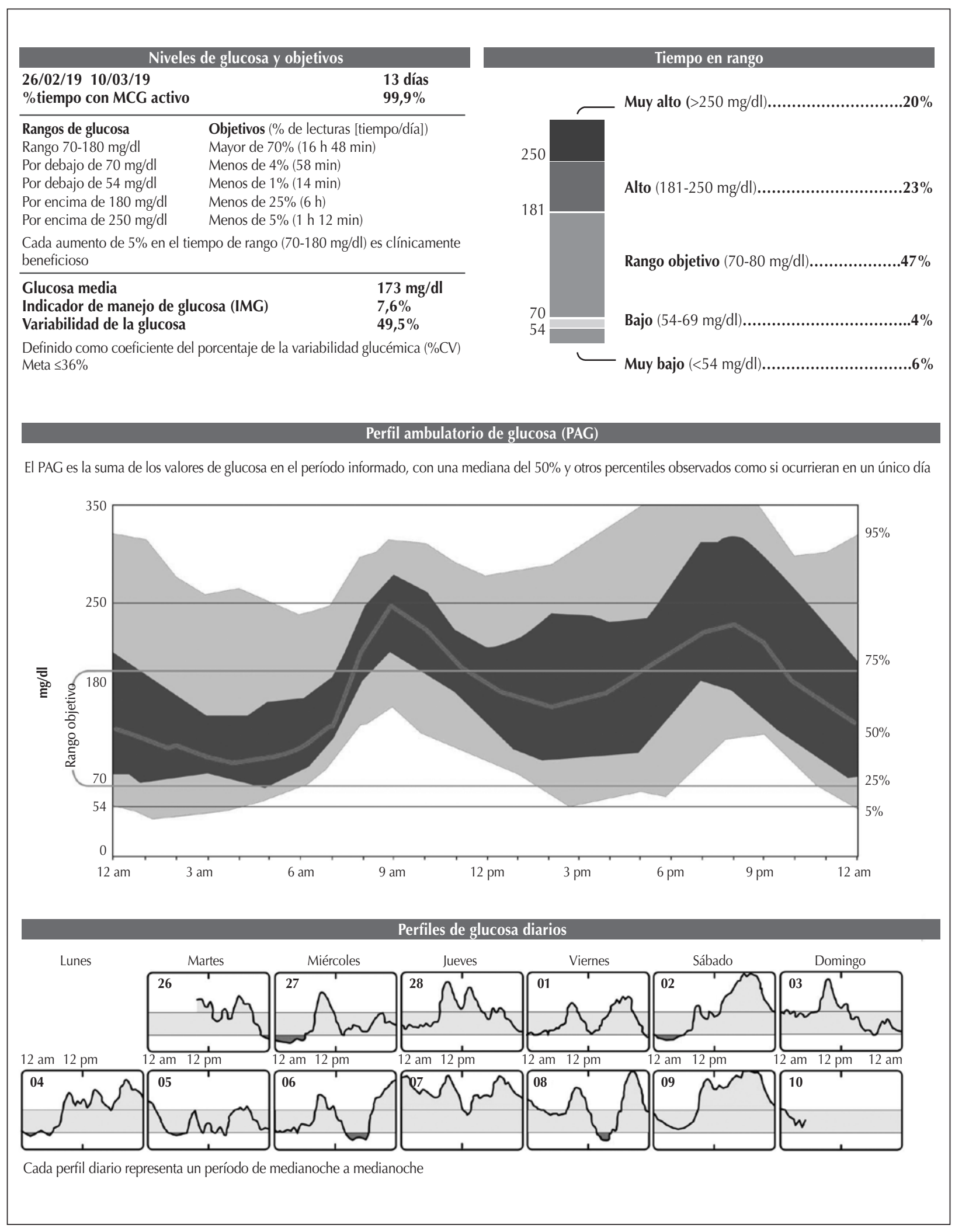

Adaptado de: Battelino et al., 2011'16.

Las cuatro métricas más relevantes: tiempo en rango de glucosa objetivo (TIR), tiempo por debajo del rango de glucosa objetivo (TBR), tiempo superior al rango de glucosa objetivo (TAR), variabilidad glucémica (\%CV).

Figura 1: Perfil ambulatorio de glucosa. 
El PAG es el informe estandarizado (Figura 1) que se muestra en una sola página con diferentes gráficos que convierten los números en imágenes para una interpretación simple, fácil y rápida. Incorpora las variables fundamentales del MCG como componente integral para la toma de decisiones clínicas ${ }^{15}$. Con ligeras modificaciones, ha sido adoptado por la mayoría de los dispositivos de MCG para realizar sus informes.

Se ha optimizado la lista de métricas (conjunto de medidas para estimar o determinar las características de un sistema de información para establecer comparaciones o planificar conductas) que selecciona las 10 variables más útiles en la práctica clínica:

1. Número de días durante los cuales se ha realizado el MCG (se recomienda considerar, al menos, 14 días) 16,17.

2. Porcentaje de tiempo en el que el sistema de MCG utilizado estuvo activo (se recomienda lograr un almacenamiento mínimo del 70\% de los datos generados en los 14 días) ${ }^{18}$. Recordar que es necesario no superar las 8 horas (h) entre una lectura y otra, lo cual obliga a realizar un mínimo de tres lecturas diarias para que se almacenen los datos en el sistema FreeStyle Libre.
3. Informe de la media de glucosa.

4. Indicador de manejo de glucosa $(\mathrm{IMG})^{19}$. Se expresa en la práctica como la HbA1c estimada por el sistema.

5. Coeficiente de variabilidad (\%CV), cuyo valor óptimo sería igual o menor del 36\%.

6. TAR nivel 2: porcentaje de lecturas y tiempo del día transcurrido con glucemias $>250 \mathrm{mg} / \mathrm{dl}$.

7. TAR nivel 1: porcentaje de lecturas y tiempo del día transcurrido con glucemias entre 181 y $250 \mathrm{mg} / \mathrm{dl}$.

8. TIR: porcentaje de lecturas y tiempo del día transcurrido dentro el rango que se determine para cada paciente (el rango consensuado es de 70-180 mg/dl, sin embargo puede variar según el perfil del paciente).

9. TBR nivel 1: porcentaje de lecturas y tiempo diario transcurrido con glucemias entre 54 y $69 \mathrm{mg} / \mathrm{dl}$.

10. TBR nivel 2: porcentaje de lecturas y tiempo diario transcurrido con glucemias $<54 \mathrm{mg} / \mathrm{dl}$.

Se describen a continuación, y a modo de sugerencia, cuáles serían los objetivos metabólicos (glucémicos principalmente) en diferentes subpoblaciones de pacientes con DM (Tabla 1).

\begin{tabular}{|c|c|c|c|c|c|c|c|c|c|}
\hline \multirow[t]{2}{*}{ Tipo de diabetes } & \multicolumn{3}{|c|}{ TIR } & \multicolumn{3}{|c|}{ TBR } & \multicolumn{3}{|c|}{ TAR } \\
\hline & $\begin{array}{l}\text { Porcentaje } \\
\text { de lecturas } \\
\text { dentro del } \\
\text { rango }\end{array}$ & $\begin{array}{c}\text { Horas diarias } \\
\text { en rango }\end{array}$ & $\begin{array}{l}\text { Objetivo } \\
\text { glucémico } \\
(\mathrm{mg} / \mathrm{dl})\end{array}$ & $\begin{array}{l}\text { Porcentaje } \\
\text { de lecturas } \\
\text { dentro del } \\
\text { rango }\end{array}$ & $\begin{array}{l}\text { Horas } \\
\text { diarias en } \\
\text { rango }\end{array}$ & $\begin{array}{l}\text { Objetivo } \\
\text { glucémico } \\
(\mathrm{mg} / \mathrm{dl})\end{array}$ & $\begin{array}{l}\text { Porcentaje } \\
\text { de lecturas } \\
\text { dentro del } \\
\text { rango }\end{array}$ & $\begin{array}{l}\text { Horas } \\
\text { diarias en } \\
\text { rango }\end{array}$ & $\begin{array}{l}\text { Objetivo } \\
\text { glucémico } \\
(\mathrm{mg} / \mathrm{dl})\end{array}$ \\
\hline $\begin{array}{l}\text { DM1 } \\
\text { DM2 } \\
\text { General }\end{array}$ & $70-180$ & $>70$ & $>16: 48 \mathrm{~h}$ & $<70<55$ & $<4<1$ & $\begin{array}{l}<01: 00 \mathrm{~h} \\
<00: 15 \mathrm{~h}\end{array}$ & $\begin{array}{l}>180 \\
>250\end{array}$ & $\begin{array}{l}<25 \\
<5\end{array}$ & $\begin{array}{l}<06: 00 \mathrm{~h} \\
<01: 12 \mathrm{~h}\end{array}$ \\
\hline Mayoresalto riesgo & $70-180$ & $>50$ & $>12: 00 \mathrm{~h}$ & $<70$ & $<1$ & $<00: 15 \mathrm{~h}$ & $>250$ & $<10$ & $<02: 24 \mathrm{~h}$ \\
\hline DM1 embarazadas & $63-140$ & $>70$ & $>16: 48 \mathrm{~h}$ & $<63<55$ & $<4<1$ & $\begin{array}{l}<01: 00 \mathrm{~h} \\
<00: 15 \mathrm{~h}\end{array}$ & $>140$ & $<25$ & $<06.00 \mathrm{~h}$ \\
\hline $\begin{array}{l}\text { DM2 embarazadas y } \\
\text { diabetes gestacional }\end{array}$ & $\mathrm{SD}$ & $\begin{array}{l}\text { El mayor } \\
\text { porcentaje } \\
\text { posible }\end{array}$ & $\begin{array}{c}\text { La mayor } \\
\text { cantidad de } \\
\text { horas posible }\end{array}$ & $<63<55$ & $\begin{array}{c}\text { El menor } \\
\text { porcentaje } \\
\text { posible }\end{array}$ & $\begin{array}{c}\text { La menor } \\
\text { cantidad de } \\
\text { horas posible }\end{array}$ & $>140$ & $\begin{array}{c}\text { El menor } \\
\text { porcentaje } \\
\text { posible }\end{array}$ & $\begin{array}{c}\text { La menor } \\
\text { cantidad de } \\
\text { horas posible }\end{array}$ \\
\hline
\end{tabular}

Adaptado de: American Diabetes Association 201920; Battelino et al., $2019^{21}$.

TIR: tiempo en rango; TBR: tiempo por debajo del rango; TAR: tiempo por encima del rango; SD: sin datos disponibles. Considerar que tanto TAR como TBR tienen dos rangos para considerar: TAR rango 1: 180-250 mg/dl; TAR rango 2: > 250 mg/dl; TBR rango 1: $55-70 \mathrm{mg} / \mathrm{dl}$ y TBR rango $2<55 \mathrm{mg} / \mathrm{dl}$. .

Tabla 1: Objetivos metabólicos. Guías para la interpretación del control de la glucosa utilizando datos de consensos internacionales.

\section{TIR (time in range o tiempo en rango)}

Los rangos delTIR deben personalizarse según las características de cada individuo, habiéndose estandarizado los siguientes objetivos del TIR (Tabla 1 y Figura 2) $)^{5}$.

- Población general de pacientes con DM1 y DM2 insulinizados: 70-180 mg/dl en más del 70\% de las lecturas del día.
- Pacientes con DM1 durante el embarazo: 63-140 mg/dl al menos 70\% de las lecturas del día.

- Pacientes con DM2 embarazadas o con diabetes gestacional: no se incluyen valores de TIR debido a la falta de evidencia sobre los objetivos de MCG en esta subpoblación.

- Pacientes lábiles o inestables: bajar el TIR del 70 al 50\% de las lecturas del día, y elTBR a menos 
del 1\% para disminuir el riesgo de hipoglucemias.

En caso de embarazo se recomienda tomar las decisiones terapéuticas complementando con los valores de glucemia capilar ${ }^{21}$.

Se ha descripto una correlación significativa entre el TIR (70-180 mg/dl) y las complicaciones crónicas de la DM, tales como progresión de retinopatía y aparición de microalbuminuria ${ }^{22,23}$. EI TIR se correlaciona con los valores de HbA1c. El 70\% del tiempo diario transcurrido entre valores glucémicos de 70 a
$180 \mathrm{mg} / \mathrm{dl}$ se correlaciona con valores de HbA1c de aproximadamente el 7\%. Por otra parte, existe una correlación entre diferentes niveles de TIR y de HbA1c. Un aumento de $10 \%$ del TIR corresponde a una disminución de la HbA1c cercana al 0,5\% (Tabla 2) 24,25.

EITIR agrega una métrica diferente y prospectiva en la evaluación del control glucémico, a diferencia de la HbA1c que aporta un dato retrospectivo de ese control (Tablas 2 y 3 ). Por lo tanto, ofrece una herramienta complementaria.

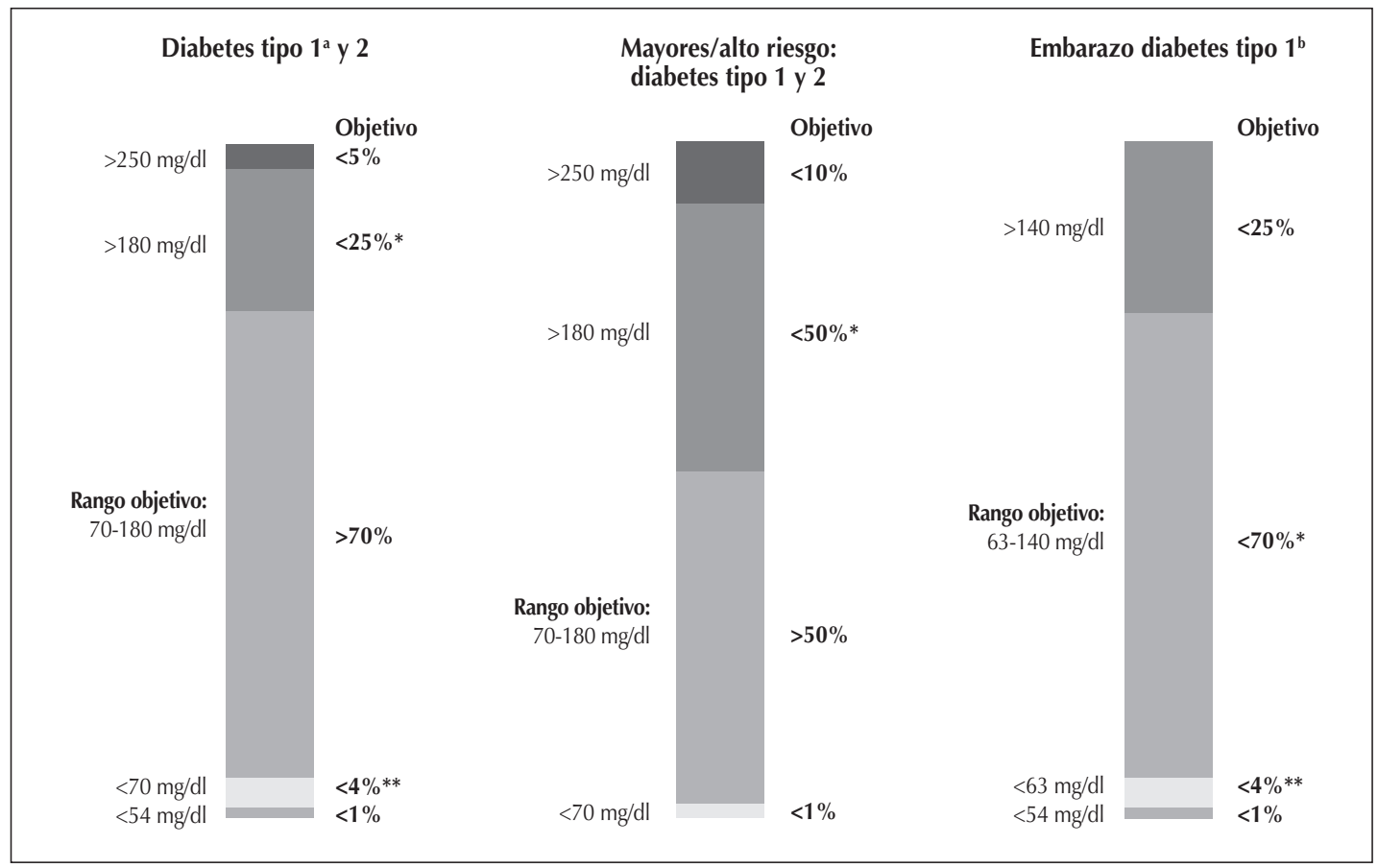

Adaptado de: Battelino et al., $2019^{16}$.

a Para <25 años. Si la meta de HbA1c 7,5\% ajuste la meta TIR a aproximadamente $60 \%$.

${ }^{b}$ Los porcentajes del tiempo en rangos están basados en evidencia limitada.

* Incluye porcentaje de valores $>250 \mathrm{mg} / \mathrm{dl}$.

* Incluye porcentaje de valores $<54 \mathrm{mg} / \mathrm{dl}$.

Figura 2: Tiempo en rango sugerido (TIR) para diferentes situaciones.

\begin{tabular}{|c|c|c|}
\hline Medición TIR & Promedio HbA1c & IC95 $\%$ \\
\hline $20 \%$ & $9,4 \%$ & 8,$0 ; 10,7 \%$ \\
\hline $30 \%$ & $8,9 \%$ & 7,$6 ; 10,2 \%$ \\
\hline $40 \%$ & $8,4 \%$ & 7,$1 ; 9,7 \%$ \\
\hline $50 \%$ & $7,9 \%$ & 6,$6 ; 9,2 \%$ \\
\hline $60 \%$ & $7,4 \%$ & 6,$1 ; 8,8 \%$ \\
\hline $70 \%$ & $7,0 \%$ & 5,$6 ; 8,3 \%$ \\
\hline $80 \%$ & $6,5 \%$ & 5,$2 ; 7,8 \%$ \\
\hline $90 \%$ & $6,0 \%$ & 4,$7 ; 7,3 \%$ \\
\hline
\end{tabular}

Adaptado de: Bergenstal et al., $2018^{19}$.

TIR: tiempo en rango; HbA1c: hemoglobina glicosilada; IC95\%: intervalo de confianza del 95\%.

Cada 10\% de incremento en TIR $=\sim 0,5 \%$ de reducción de $A 1 C$.

\begin{tabular}{|l|l|}
\hline \multicolumn{1}{|c|}{ HbA1c } & \multicolumn{1}{c|}{ Tiempos en rango } \\
\hline Evalúa niveles únicos de HbA1c & $\begin{array}{l}\text { Evalúa niveles } \\
\text { continuos de glucosa }\end{array}$ \\
\hline $\begin{array}{l}\text { Compara parámetros de } \\
\text { glicosilación cada tres meses } \\
\text { retrospectivamente }\end{array}$ & $\begin{array}{l}\text { Compara fluctuaciones } \\
\text { continuamente y } \\
\text { prospectivamente }\end{array}$ \\
\hline No captura hipoglucemias & $\begin{array}{l}\text { Captura todos los niveles de } \\
\text { glucemia en un rango de tiempo }\end{array}$ \\
\hline $\begin{array}{l}\text { Menos probable que capture el } \\
\text { impacto de intervenciones agudas }\end{array}$ & $\begin{array}{l}\text { Probable que capture el impacto } \\
\text { de intervenciones agudas }\end{array}$ \\
\hline
\end{tabular}

TIR: tiempo en rango; HbA1c: hemoglobina glicosilada.

Tabla 3: Diferencias en la información obtenida con TIR (tiempo en rango) vs $\mathrm{HbA} 1 \mathrm{c}$.

Tabla 2: Correlación de HbA1c con tiempo en rango. 


\section{Coeficiente de variabilidad}

La evaluación de la variabilidad glucémica es otra de las métricas del PAG que completa las mediciones más relevantes del MCG. Se calcula mediante el \% $\mathrm{CV}^{26}$, considerando como objetivo terapéutico lograr que sea $\leq 36 \%$. Un $\%$ CV por encima de este valor se asocia con mayor frecuencia de hipoglucemias ${ }^{27}$. Objetivos de \%CV más bajos $(<33 \%)$ brindan protección adicional contra la hipoglucemia para los pacientes que reciben tratamiento con insulina o sulfonilureas ${ }^{28}$.

El cálculo del \%CV se realiza al dividir la desviación estándar por la glucemia media x 100 (SD/ media $\times 100)^{29}$.

Para la interpretación adecuada del MCG es necesario considerar las métricas del PAG en diferentes momentos del día. Para ello, es aconsejable dividir las $24 \mathrm{~h}$ del día en los siguientes períodos para interpretar los resultados adecuadamente y luego modificar el tratamiento para mejorar las glucemias en dichos períodos.

\section{Períodos en relación con la ingesta (Figura 3)}

1. Período nocturno temprano o de posabsorción de la cena (00:00 a 04:00 h). Es la primera parte de la noche y está impactado por los efectos de la ingesta y la dosis de insulina prandial de la pre cena.

2. Período nocturno tardío (verdadero período de ayuno) (04:00 a 08:00 h). Es la segunda parte de la noche y está impactado por la dosis y los efectos de la insulina basal, el efecto residual de la actividad física del día anterior y la magnitud del fenómeno del alba.

3. Períodos prepandiales ( 30 minutos antes de cada una de las ingestas del día y momento habitualmente utilizado para controlar el nivel de glucosa y aplicar insulina prandial). Este período está influenciado por los efectos de la insulina prandial de la comida previa (si ocurrió entre 2 y 4 h antes), los efectos de la insulina basal residual y la prolongación del período de posabsorción de la ingesta anterior.

4. Períodos prandiales, llamados también postprandiales (hasta 2 a $3 \mathrm{~h}$ luego de la ingesta), afectados por los efectos de la ingesta de alimentos y la acción de la insulina prandial.

5. Períodos de posabsorción o posabsortivos (entre 3 y $8 \mathrm{~h}$ posteriores a la ingesta). Impactados por los efectos de las ingestas, la dosis de insulina prandial previa, la insulina basal residual circulante, la prolongación de los períodos posabsortivos, la actividad física y el tiempo transcurrido sin ingerir alimentos.

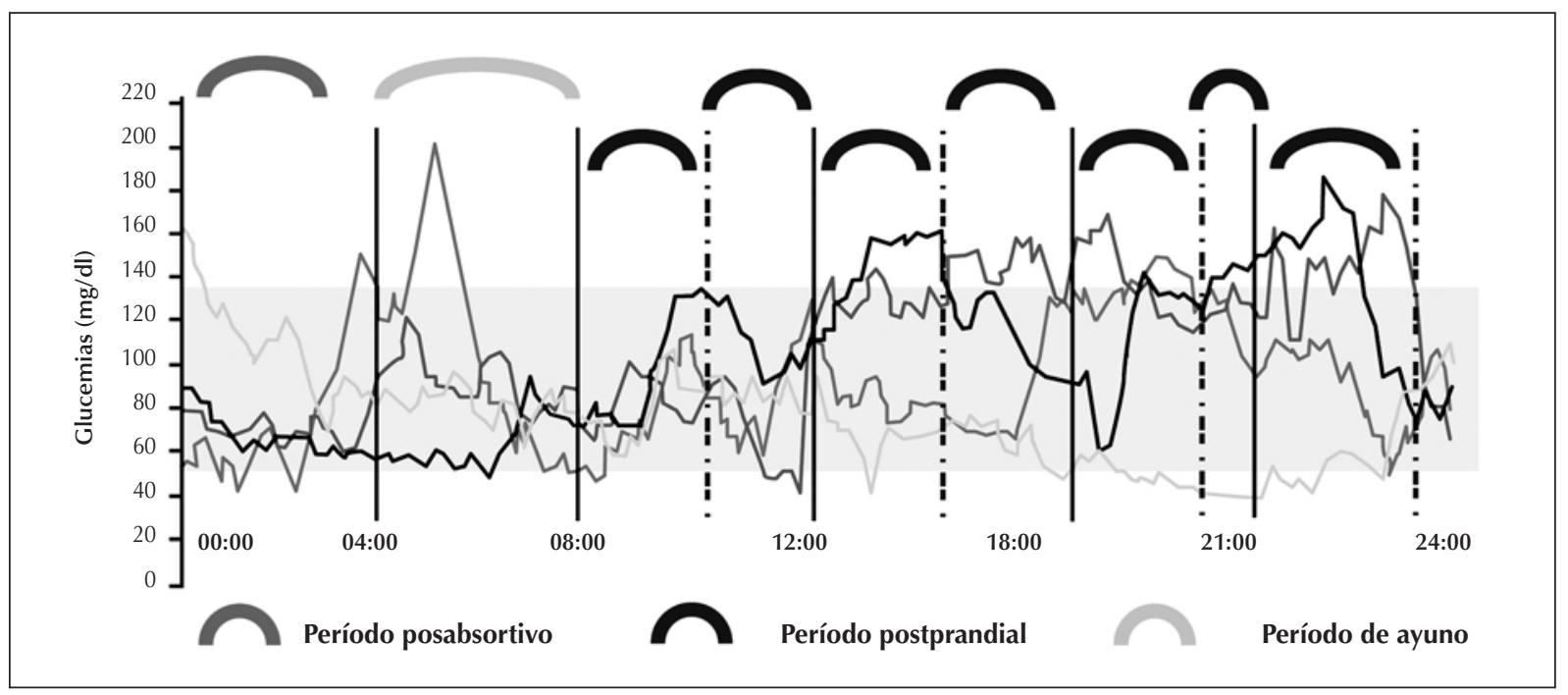

El período prandial (llaves negras) es el lapso entre el comienzo de la ingesta y las 2 a 3 h subsiguientes. El período de posabsorción (llaves gris oscuro) comprende entre las 3 h siguientes a una ingesta y la próxima comida. El período de ayuno verdadero (llaves gris clarol es el período entre las 2 y 3 h de la madrugada y el desayuno ${ }^{32}$.

Figura 3: Monitoreo glucémico en personas sin diabetes en relación con la ingesta. 


\section{Períodos del día más adecuados para interpretar el MCG}

\section{¿Cómo evaluar la dosis de insulina basal?}

Para ello es necesario identificar los momentos de ayuno alimentario más prolongados (al menos 8 h). Puede ser alguno de los siguientes:

- Período nocturno tardío. Ideal para evaluar la dosis basal durante la noche (sobre todo la segunda parte de ésta). Se recomienda seleccionar el día con el menor nivel de glucosa como el momento ideal para hacer los ajustes en la dosis de insulina basal nocturna para que, una vez hechas las modificaciones, se eviten las hipoglucemias (Figura 4).
- Período diurno en que el paciente saltea alguna comida (en esta situación el período entre una ingesta y otra posiblemente sea de 8 o más horas). Es frecuente observar este escenario en pacientes que habitualmente saltean la merienda y, por lo tanto, no ingieren comida entre el almuerzo y la cena. Éste sería un momento ideal para evaluar la dosis basal durante el día. También se sugiere buscar el día con el menor nivel de glucosa intersticial como el más adecuado para hacer los ajustes en la dosis basal diurna a fin de evitar las hipoglucemias (Figura 5).

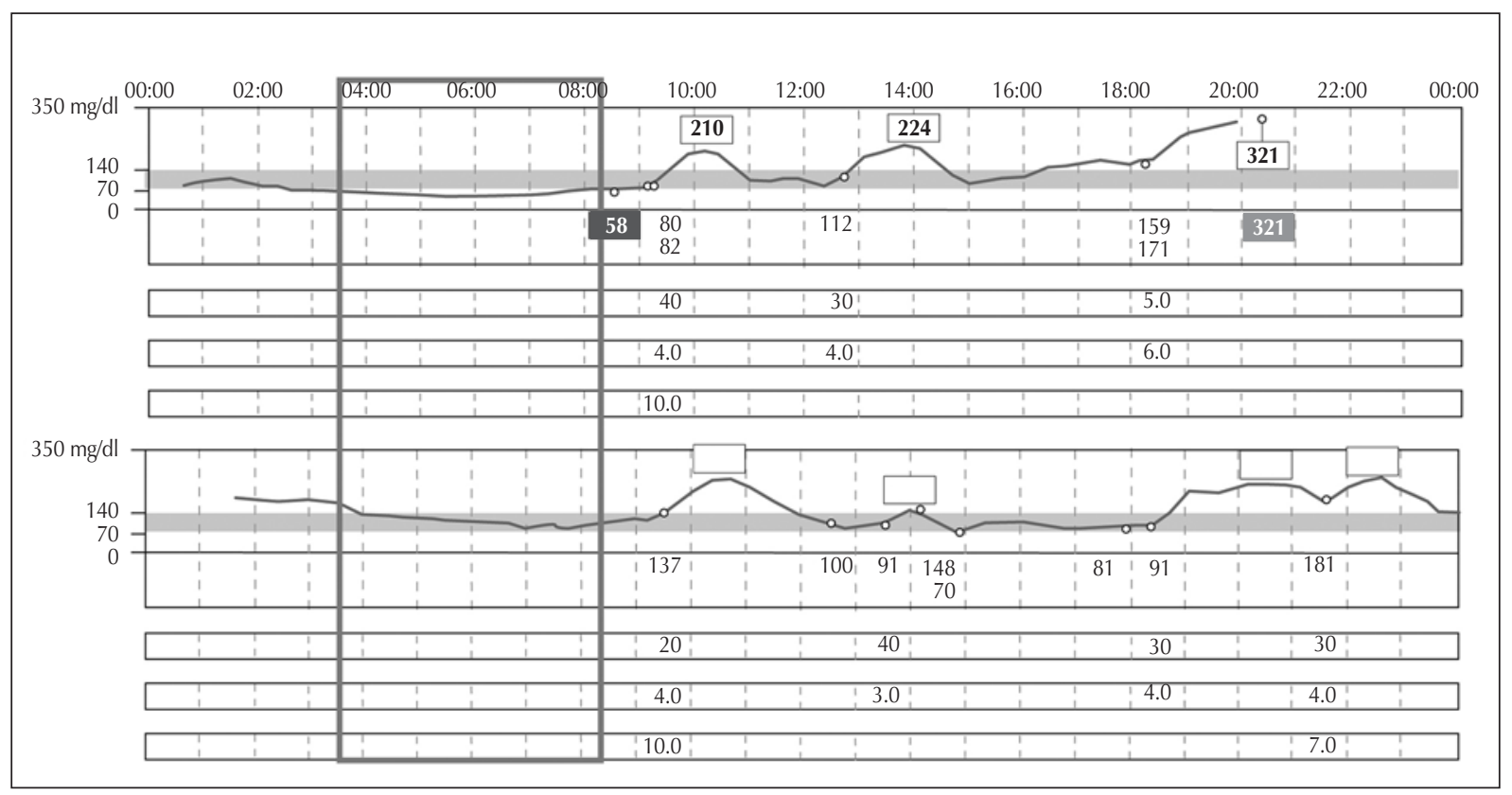

Adaptado de: Xing et al., 201118; Riddlesworth et al., $2018^{19}$ y Litwak et al., $2019^{32}$.

Figura 4: Período nocturno tardío. Momento adecuado para evaluar dosis de insulina basal aplicada en la noche.

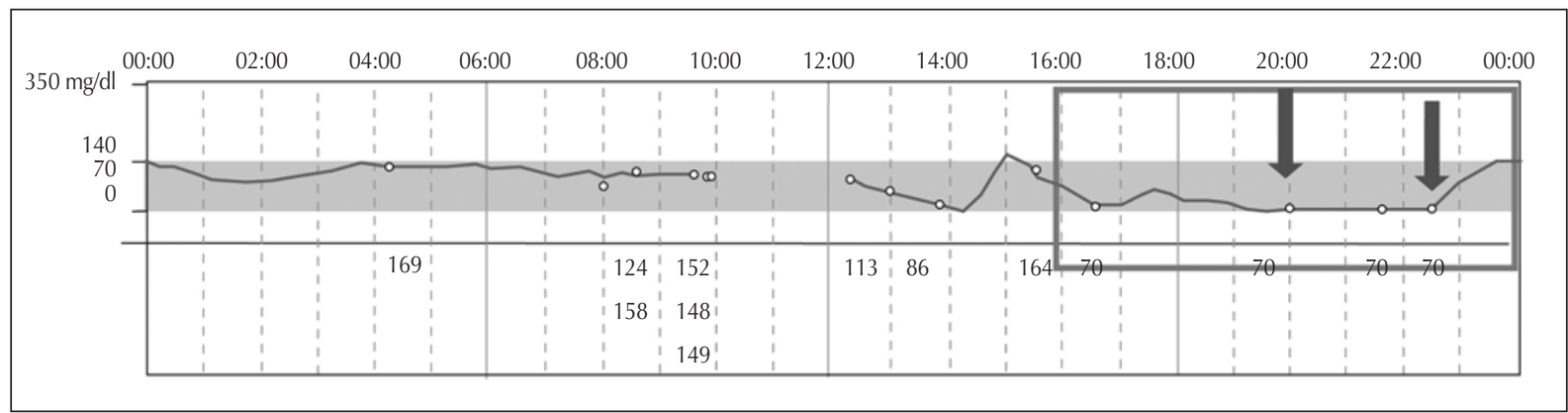

Adaptado de: Xing et al., 2011;18 Riddlesworth et al., 2018,19 y Litwak et al., $2019^{32}$.

Figura 5: Período diurno de ayuno alimentario por salteo de una ingesta o por haber prolongado el tiempo de ingesta entre una comida y otra. Momento adecuado para evaluar la dosis de insulina basal aplicada durante el día. 


\section{¿Cómo evaluar la dosis de insulina prandial?}

Para evaluar y corregir las dosis prandiales de insulina es necesario considerar, por una parte, la relación insulina/gramos de carbohidratos (CHD), y por otra, el índice de sensibilidad a la insulina. A estos dos parámetros clásicamente considerados en el AGC, se agrega un tercer componente que brinda sólo el MCG. Es la tendencia que tendrá la glucosa en los próximos 30 minutos, calculada en función de la evolución de la glucosa intersticial analizada retrospectivamente y del historial de las últimas horas. Se expresa por un código de flechas en la pantalla (ver más adelante).

- Relación (ratio, en inglés) dosis insulina/gramos de carbohidratos (I/CHD). Es la cantidad de insulina necesaria para metabolizar una determinada cantidad de gramos de carbohidratos. Se evalúa analizando el efecto de una dosis predeterminada de insulina de acción rápida (por ejemplo, 1 unidad cada 5 a $20 \mathrm{~g}$ de $\mathrm{CHD}$, expresada como I/CHD: 1/5, $1 / 10,1 / 15$, etc.). Se aplica antes de una ingesta en la cual la glucosa se encuentre dentro de un rango adecuado (idealmente entre 70 y 130 mg/dl). Su efecto se evalúa entre los 90 y 120 minutos postprandiales. Se considera que la dosis fue adecuada si se logran niveles entre 140 y 160 mg/dl idealmente (evitando que supere los $180 \mathrm{mg} / \mathrm{dll}$ ). Se sugiere hacer estos ajustes en ingestas que contengan entre 40 y $60 \mathrm{~g}$ de CHD (evitar que superen los $80 \mathrm{~g}$ de CHD por comida) (Figura 6).

- Índice de sensibilidad a la insulina (IS). Debería analizarse en los momentos en los que el paciente realiza correcciones no relacionadas con una ingesta y al menos $4 \mathrm{~h}$ después de una dosis de insulina de acción rápida para evitar la presencia de insulina activa. Entonces se puede calcular en cuánto puede descender el nivel de glucosa una unidad de insulina de acción rápida (por ejemplo, una unidad desciende 20, 30, 50 o más mg/dl el nivel de glucosa). Sobre la base de estas observaciones puede establecerse el IS en: 1/20, 1/30 o 1/50. El IS inicial se calcula dividiendo 1.800 por la dosis total de insulina (1.800/DTI). Por ejemplo, si un paciente recibe en total 60 unidades de insulina por día, su IS es $30(1.800 / 60=30)$, o sea que requiere una unidad de insulina para disminuir $30 \mathrm{mg}$ de glucemia.

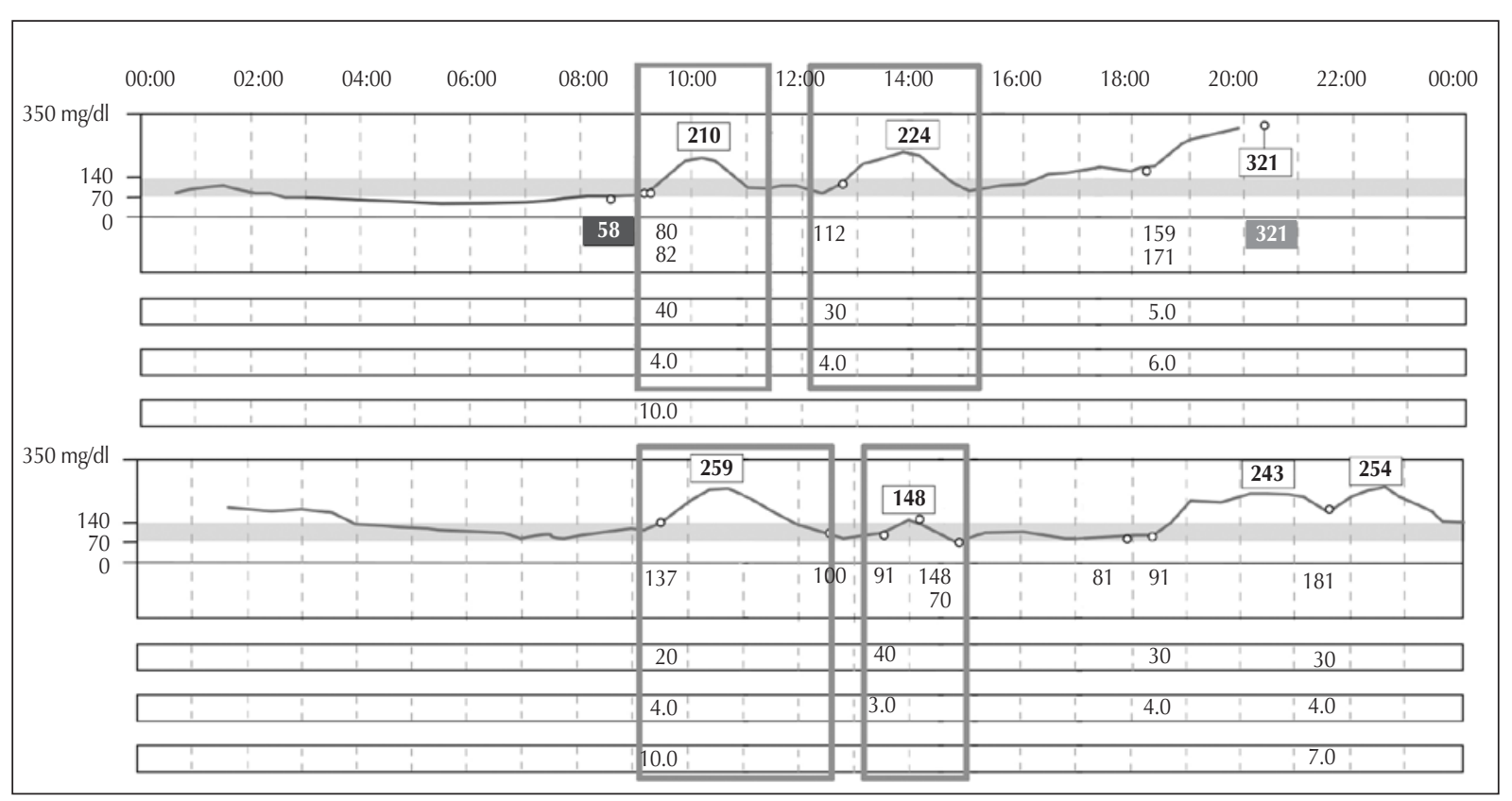

Adaptado de: Xing et al., 2011,18; Riddlesworth et al., 2018,19 y Litwak et al., $2019^{32}$.

Figura 6: Evaluación de la relación I/CHD frente a ingestas que comienzan en un nivel glucémico adecuado con el objetivo de determinar el efecto de la insulina sobre el contenido de CHD.

\section{Sugerencias de conductas terapéuticas}

Al margen de la disponibilidad continua de datos, uno de los aportes más importantes e innovadores del MCG es la posibilidad de conocer la tendencia de las glucemias expresada en forma de flechas (flechas de tendencia). Las modificaciones del tratamiento que consideren al mismo tiempo los niveles de glucosa y las tendencias 
son aún controvertidas, dado que no hay evidencia suficiente que indique cuál es la mejor opción para tomar decisiones con criterios terapéuticos. Sin embargo, existen consensos internacionales que sugieren diferentes formas de calcular tanto las dosis de insulina como los aportes de carbohidratos en diferentes momentos del día sobre la base de los niveles de glucosa y sus tendencias. Como orientación frente a la toma de decisiones, pueden considerarse tres situaciones:

1. Realización de ajustes terapéuticos según el valor glucémico y las tendencias en el período prepandial en adultos

En el MCG, el dato de glucosa obtenido antes de una comida se acompaña por la tendencia que, tal como se describió previamente, estima el nivel glucémico que tendrá el paciente en los próximos 30 minutos en función de la glucosa evaluada en forma retrospectiva y en relación con el historial de las últimas horas. Esta se expresa mediante fle- chas en la pantalla del lector. Por lo tanto, a diferencia del cálculo de dosis que se efectúa con el AGC tradicional que considera sólo glucemia y gramos de CHD a ingerir), en el caso del MCG la dosis de insulina prandial puede decidirse también en función de la tendencia, agregando o disminuyendo la dosis del bolo prepandial según la tendencia de la glucosa (Tabla 4). Sólo a modo de ejemplo, si antes de una ingesta regular (desayuno, almuerzo, merienda o cena) se observa una flecha que apunta hacia arriba $\left(90^{\circ}\right)$ en el equipo MCG-IS/Flash o bien 2 a 3 flechas verticales en los equipos MCG-RTS junto con niveles de glucosa elevados (>250 mg/dl), se debería agregar 1 a 3 unidades de insulina al cálculo inicial realizado sobre la base del I/CHD más IS. Por el contrario, si la flecha muestra glucemias bajas $(<100 \mathrm{mg} / \mathrm{dl})$ asociado con una tendencia hacia abajo, ya sea $45^{\circ}$ o $90^{\circ}$ (según los equipos utilizados), habría que restar 1 a 2 unidades de insulina al cálculo inicial respectivamente (Tabla 4) $)^{3,13-33}$.

\begin{tabular}{|c|c|c|c|c|c|}
\hline \multicolumn{6}{|c|}{ Período prepandial adultos } \\
\hline \multirow{2}{*}{ Valor de glucosa } & \multirow{2}{*}{ MCG-IS/Flash } & \multicolumn{3}{|c|}{ MCG-RTS } & \multirow{2}{*}{ Conducta } \\
\hline & & Dexcom G5-G6 & Guardian & Eversense & \\
\hline$<250 \mathrm{mg} \%$ & $\rightarrow$ o $\nearrow$ & $\rightarrow$ o $\nearrow$ & $\uparrow$ & $\rightarrow$ o $\nearrow$ & Corregir valor absoluto (FS) \\
\hline$>250 \mathrm{mg} \%$ & $\uparrow$ & $\begin{array}{c}\uparrow-\uparrow \uparrow \\
\end{array}$ & 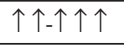 & $\uparrow$ & $+1 \mathrm{u}$ \\
\hline$>300 \mathrm{mg} \%$ & $\uparrow$ & $\begin{array}{l}\uparrow-\uparrow \uparrow \\
\end{array}$ & 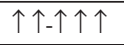 & $\uparrow$ & $+2 u$ \\
\hline$>350 \mathrm{mg} \%$ & $\uparrow$ & $\begin{array}{l}\uparrow-\uparrow \uparrow \\
\end{array}$ & 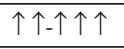 & $\uparrow$ & $+3 u$ \\
\hline Cercano a $100 \mathrm{mg} \%$ & $\downarrow$ & $\downarrow \downarrow \downarrow \downarrow$ & $\downarrow \downarrow \downarrow-\downarrow \downarrow \downarrow$ & $\downarrow$ & $-2 U$ \\
\hline Cercano a $100 \mathrm{mg} \%$ & $\searrow$ & $\searrow-\downarrow$ & $\downarrow$ & $\searrow$ & $-1 U$ \\
\hline
\end{tabular}

MCG-IS/Flash: monitoreo de glucosa intermitente; MCG-RTS: monitoreo continuo de glucosa en tiempo real. Guardian y Eversense: sistemas de MCG-RTS que no se encuentran en Argentina.

Tabla 4: Ajustes terapéuticos según las tendencias y el valor glucémico en el período prepandial en adultos.

2. Realización de ajustes terapéuticos según el valor glucémico y las tendencias en los períodos postprandial y de posabsorción en adultos

Se recomienda no realizar bolos correctivos dentro de las 3 a $4 \mathrm{~h}$ posteriores al último bolo de insulina regular o análogo rápido, considerando que la insulina inyectada previamente sigue aún activa. Se debe asegurar que la hiperglucemia que se corregirá no haya sido consecuencia de la corrección a una hipoglucemia previa (hiperglucemia inducida). Por lo tanto, no administrar insulina dentro de las $3 \mathrm{~h}$ de la inyección de insulina rápida previa y sólo hacerlo si se considera que la hiperglucemia no es reactiva a una hipoglucemia. Si se observa que las dosis prandiales son insuficientes en forma repetida, debe reevaluarse el IS del paciente para modificar y adecuar (aumentar) la dosis de los futuros bolos (Tabla 5). 


\begin{tabular}{|c|c|c|c|c|c|}
\hline \multicolumn{6}{|c|}{ Período postprandial adultos } \\
\hline \multirow{2}{*}{ Valor de glucosa } & \multirow{2}{*}{ MCG-IS/Flash } & \multicolumn{3}{|c|}{ MCG-RTS } & \multirow{2}{*}{ Conducta } \\
\hline & & Dexcom G5-G6 & Guardian & Eversense & \\
\hline$>$ & $\rightarrow$ o $\nearrow$ & $\pi$ & $\uparrow$ & $\rightarrow$ o $\nearrow$ & Corregir valor absoluto \\
\hline$>200 \mathrm{mg} \%$ & $\uparrow$ & 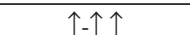 & 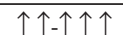 & $\uparrow$ & 1 unidad \\
\hline$>250 \mathrm{mg} \%$ & $\uparrow$ & 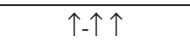 & $\overline{\imath \uparrow \uparrow-\uparrow \uparrow \uparrow}$ & $\uparrow$ & 2 unidades \\
\hline$>300 \mathrm{mg} \%$ & $\uparrow$ & 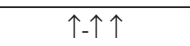 & 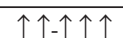 & $\uparrow$ & 3 unidades \\
\hline$>350 \mathrm{mg} \%$ & $\uparrow$ & 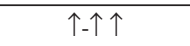 & 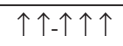 & $\uparrow$ & 4 \\
\hline Cercano a $100 \mathrm{mg} \%$ & 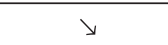 & $\searrow-\downarrow$ & $\downarrow$ & 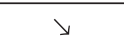 & Ingerir 10 g carbohidratos \\
\hline Cercano a $100 \mathrm{mg} \%$ & $\downarrow$ & $\downarrow-\downarrow \downarrow$ & $\downarrow \downarrow-\downarrow \downarrow \downarrow$ & $\downarrow$ & Ingerir $15 \mathrm{~g}$ \\
\hline
\end{tabular}

MCG-IS/Flash: monitoreo de glucosa intermitente; MCG-RTS: monitoreo continuo de glucosa en tiempo real. Guardian y Eversense: sistemas de MCG-RTS que no se encuentran en Argentina.

Tabla 5: Ajustes terapéuticos según las tendencias y el valor glucémico en el período postprandial en adultos con un IS estandarizado en un promedio de 1/50.

3. Realización de ajustes terapéuticos según el valor glucémico y las tendencias en niños y adolescentes en el período prepandial ${ }^{10}$

Dadas las diferencias entre los grupos etarios se sugiere efectuar el cálculo de dosis para los ajustes sobre la base del IS en pacientes pediátricos y adolescentes (Tabla 6). Se debe considerar el tiempo de latencia de la insulina regular o de los análogos de acción rápida. Por lo tanto, si el lector de glucosa muestra flechas con tendencia hacia arriba en el período prepandial, es necesario anticipar el momento de la inyección en más de 15 a 30 minutos, aumentar un $10 \%$ la dosis calculada del bolo si la flecha está a $45^{\circ}$, y $20 \%$ si está a $90^{\circ}$ aproximadamente. En cambio, si el lector muestra flechas con tendencias hacia abajo se debe administrar la insulina prácticamente en el momento del consumo de los alimentos: reducir $10 \%$ la dosis si la flecha tiene una tendencia hacia abajo de $45^{\circ}$, y $20 \%$ si la tendencia es de $90^{\circ}$ aproximadamente.

\begin{tabular}{|c|c|c|}
\hline Medición TIR & & IS \\
\hline Prescolares 2-6 años & Mayor sensibilidad & $125 \mathrm{mg} / \mathrm{dL}$ \\
\hline Escolares 7-12 años & Gran variabilidad & $\geq 50 \mathrm{a}<75 \mathrm{mg} / \mathrm{dL}$ \\
\hline Adolescentes/adultos jóvenes 13-22 años & & 25,50 ó $<25 \mathrm{mg} / \mathrm{dL}$ \\
\hline
\end{tabular}

Adaptado de: Laffel et al., $2017^{10}$

Considerar según la etapa de desarrollo del paciente los diferentes IS para calcular en cuánto descendería la glucosa con una unidad de insulina de acción rápida. IS, índice de sensibilidad a la insulina, que expresa cuántos mg de glucosa bajarían una unidad de insulina. Los valores de IS son aproximados. Dada la gran variabilidad en estos grupos etarios en relación con la sensibilidad, se sugiere realizar el cálculo individualizado en relación con el peso con la fórmula previamente citada en el texto, de 1800 dividido en la dosis total de insulina -1.800/DTI (esta última calculada por peso 0,5-0,7 U/kg total, con el fin de minimizar los riesgos de hipoglucemias). TIR: tiempo en rango; IS: índice de sensibilidad a la insulina.

Tabla 6: Insulinosensibilidad en niños y adolescentes.

Como medida de precaución, se sugiere que los controles de glucosa antes de dormir deberían estar alrededor del objetivo $-130 \mathrm{mg} / \mathrm{dl}-$, con una tendencia horizontal $(\rightarrow)$ o hacia arriba $(\lambda)$ a $45^{\circ}$. Si es necesario hacer ajustes con insulina, se propone efectuarlos con mucha prudencia (Tabla 7).

\begin{tabular}{|c|c|c|c|c|c|c|c|}
\hline Valor de glucosa & MCG-IS/Flash & \multicolumn{3}{|c|}{ MCG-RTS } & \multicolumn{3}{c|}{ IS } \\
\hline & & Dexcom G5-G6 & Guardian & Eversense & Preescolar $\geq 125$ & Escolar $\geq 50$ a $<75$ & Adolescente 25 -50 \\
\hline$<250 \mathrm{mg} \%$ & $\uparrow$ & $\uparrow-\uparrow \uparrow$ & $\uparrow \uparrow-\uparrow \uparrow \uparrow$ & $\uparrow$ & $+0,5$ unidades & +1 unidad & +1 a 2 unidades \\
\hline Cercano a $100 \mathrm{mg} \%$ & $\downarrow$ & $\downarrow-\downarrow \downarrow$ & $\downarrow \downarrow-\downarrow \downarrow \downarrow$ & $\downarrow$ & $\downarrow$ dosis $20 \%$ & $\downarrow$ dosis $20 \%$ & $\downarrow$ dosis $20 \%$ \\
\hline Cercano a $100 \mathrm{mg} \%$ & $\searrow$ & $\searrow-\downarrow$ & $\downarrow$ & $\searrow$ & $\downarrow$ dosis $10 \%$ & $\downarrow$ dosis $10 \%$ & $\downarrow$ dosis $10 \%$ \\
\hline
\end{tabular}

Adaptado de: Laffel et al., $2017^{10}$.

MCG-IS/Flash: monitoreo de glucosa intermitente; MCG-RTS: monitoreo continuo de glucosa en tiempo real; IS: índice de sensibilidad a la insulina.

Eversense: no se encuentra en Argentina.

Tabla 7: Conductas terapéuticas sugeridas según el valor de glucosa y las tendencias en niños y adolescentes. 


\section{Efectos secundarios indeseables del MCG}

Aunque no son efectos graves, generan habitualmente la suspensión de la utilización del MCG. Se han informado cuadros de dermatitis de contacto producidos por los sensores con cualquiera de los dispositivos comercializados. En algunos casos, esto se ha relacionado con la presencia de acrilato de isobornilo, que puede sensibilizar la piel y causar una reacción alérgica. Pueden realizarse pruebas de parche para identificar la causa de la dermatitis de contacto. Por otra parte, la inadecuada elección del sitio para colocar el sensor puede producir el desprendimiento de este último, al igual que la excesiva transpiración y la permanencia prolongada en el agua ${ }^{4}$.

\section{Aspectos a considerar y limitaciones del monitoreo continuo de glucosa -real time- y del monitoreo continuo -IS flash-}

- Se requiere continuar con AGC para calibrar diariamente alguno de los equipos (sistemas de Dexcom y Medtronic), aunque esto no es necesario en los equipos MCG-IS/Flash que vienen calibrados de fábrica (Sistema FreeStyle Libre). Es importante realizar un control capilar cuando existan dudas del valor aportado por el MCG o frente a síntomas que sugieren hipoglucemia.

- Puede haber diferencias episódicas en el rendimiento del sensor en la misma persona.

- Los sensores tienen un tiempo de utilización determinado.

- Las diferencias entre los datos de glucosa intersticial y capilar son más pronunciadas durante los períodos de cambio rápido en la glucemia, como los períodos prandiales o post ejercicio, debido al tiempo de retardo de medición de los equipos y al impacto del ejercicio en los diferentes compartimentos orgánicos ${ }^{34,35}$.

\section{Aportes y utilización de las plataformas digitales ${ }^{36}$ \\ - Plataforma LibreView}

LibreView es un sistema de control de la diabetes al que se ingresa desde una plataforma online que permite obtener informes de valor que ayudan a tomar mejores decisiones sobre los tratamientos. Dentro de los informes disponibles se encuentran: a) reporte AGP: reporte basado en el último consenso TIR publicado ${ }^{14}$ que incluye información sobre estadísticas y objetivos de glucosa, tiempo en rango, perfil ambulatorio de glucosa, coeficiente de variación y perfiles diarios de glucosa; b) instantánea: es un resumen rápido de los datos de glucosa; c) visualización del patrón de glucosa; d) resumen mensual, semanal y diario; e) patrones de comidas y patrones diarios.

Parte del valor agregado de la plataforma se basa en que los pacientes pueden compartir de forma remota la información de sus mediciones de glucosa con sus profesionales de la salud y la posibilidad de éstos para crear "centros/consultorios" donde compartir la información de sus pacientes con otros miembros del equipo médico.

\section{CONCLUSIONES}

EI MCG constituye, hoy en día, una herramienta muy útil y complementaria a lo que aportan la HbA1c y el AGC para el tratamiento de pacientes con DM1 y DM2 en insulinoterapia, sean niños, adolescentes o adultos. Si bien la experiencia es aún restringida, el uso del MCG, ya sea con MDI o con BPI, se asocia con descensos significativos en la HbA1c, disminución de la variabilidad glucémica, reducción de hipoglucemias totales y nocturnas, y mejoría de la calidad de vida en estos pacientes ${ }^{4-20}$.

EI MCG introdujo nuevas métricas para el control glucémico5. El PAG brindado por el MCG, cuyos principales componentes son el TIR, el TBR y el TAR, permite leer el valor de glucosa intersticial en forma rápida, observar mediante flechas las tendencias de los niveles de glucosa, las desviaciones estándar, las oscilaciones glucémicas y su variabilidad. Todos estos datos y patrones pueden observarse en diferentes lapsos de tiempo, desde períodos de 8 y 24 h, y de 7, 14, 30 y 90 días, lo que permite evaluar en forma muy dinámica la evolución del control glucémico en función de las conductas terapéuticas adoptadas y en relación con los factores intercurrentes. Dado que el MCG es una herramienta novedosa, es muy importante adquirir el entrenamiento necesario para obtener el máximo de los resultados posible.

En ese sentido, esta recomendación de expertos podría considerarse como una guía inicial para nuestro medio, teniendo en cuenta cuáles serían las indicaciones del MCG, cómo debería efectuarse la lectura e interpretación de los datos contenidos en el PAG, y cuáles serían los objetivos glucémicos en diferentes poblaciones de pacientes con diabetes.

A modo de síntesis, y considerando las nuevas métricas descriptas, surgen de diferentes consensos y recomendaciones los siguientes objeti- 
vos terapéuticos para la mayoría de los pacientes con DM1 y DM2 en insulinoterapia: TIR entre 70 y $180 \mathrm{mg} / \mathrm{dl}$ durante más del $70 \%$ del día, con un TBR menor de $70 \mathrm{mg} / \mathrm{dl}$ durante menos del $5 \%$ del día (no más del 1\% en menos de $55 \mathrm{mg} / \mathrm{dl}$ ) y un TAR mayor de $180 \mathrm{mg} / \mathrm{dl}$ durante menos del $25 \%$ del día (cuidando que no más del $5 \%$ de ese total supere los $250 \mathrm{mg} / \mathrm{dll})^{5,37-39}$. Sin embargo, es necesario individualizar los objetivos terapéuticos y considerar cuáles deberían ser estas metas en poblaciones especiales como pacientes mayores o con múltiples factores de riesgo, como también para la población pediátrica y las embarazadas. Es importante considerar que todo incremento del 10\% del TIR se acompaña, en promedio, con un descenso en la HbA1c del 0,5\% ${ }^{24,25}$. Estas métricas deberán utilizarse para establecer las dosis adecuadas, tanto de insulina basal como las dosis de los bolos. Además, sobre la base de estos datos puede establecerse mejor el IS a la insulina y determinar cuáles son los momentos del día en los que es mayor o menor el CV y buscar las causas de ello. Futuras investigaciones modificarán y ajustarán estos nuevos parámetros de control metabólico e introducirán nuevas características y funciones en el MCG con la finalidad de obtener mayores beneficios en el tratamiento de los pacientes con $\mathrm{DM}^{37}$.

\section{GLOSARIOY ABREVIATURAS}

Hemos decidido respetar las siglas en inglés para que, de esta manera, podamos comparar con otras guías internacionales los conceptos y métricas del presente trabajo.

AGC: automonitoreo glucémico capilar.

PAG: perfil ambulatorio de glucosa.

MCG: monitoreo continuo de glucosa.

MCG-RTS sistema de monitoreo continuo de glucosa en tiempo real.

MCG-IS/Flash: sistema de monitoreo continuo de glucosa de lectura intermitente (flash).

TII: terapéuticas insulínicas inyectables.

BPI: bomba portable de insulina.

ICG: indicador del control glucémico ( $\mathrm{HbA} 1 \mathrm{c}$ estimada por el sistema).

TIR: tiempo en rango.

TAR: tiempo superior al rango.

TAR nivel 1: tiempo superior al rango con niveles de glucosa entre 180 y $250 \mathrm{mg} / \mathrm{dl}$.

TAR nivel 2: tiempo superior al rango con niveles de glucosa $>250 \mathrm{mg} / \mathrm{dl}$.

TBR: tiempo por debajo del rango.
TBR nivel 1: tiempo por debajo del rango con niveles de glucosa entre 55 y $70 \mathrm{mg} / \mathrm{dl}$.

TBR nivel 2: tiempo por debajo del rango con niveles de glucosa $<55 \mathrm{mg} / \mathrm{dl}$.

IS: índice de sensibilidad a la insulina.

I/CHD: relación dosis de insulina/gramos de carbohidratos.

\section{BIBLIOGRAFÍA}

1. American Diabetes Association. Glycemic targets: Standards of medical care in diabetes-2020. Diabetes Care 2020 Jan; 43(Supplement 1):S66-S76.

2. Litwak LE, Mileo VR, Álvarez A, Gutman RA. Autocontrol de la glucemia capilar: evaluación del resultado a largo plazo (3 a 7 años). Medicina (Buenos Aires) 1999; 59:71-78.

3. Commendatore VF, Linari MA, Dieuzeide G, Ferraro M, Lapertosa S, Puchulu F, et al. Automonitoreo y monitoreo de glucosa y cetonas en la persona con diabetes. Rev Soc Arg Diab 2007; 41:95-104.

4. American Diabetes Association. Diabetes technology: Standards of medical care in diabetes 2020. Diabetes Care 2020; S77-S88.

5. Advani A. Positioning time in range in diabetes management. Diabetologia 2020; 63:242-252.

6. U.S. Food and Drug Administration. FreeStyle Libre Flash Glucose Monitoring System: summary of safety and effectiveness data (SSED). Disponible en:. https://www.accessdata.fda. gov/scripts/cdrh/cfdocs/cfpma/pma.cfm?id=P160030. Acceso: 06/07/2017.

7. Bailey TS, Grunberger G, Bode BW, Handelsman Y, Hirsch IB, Jovanovic $L$, et al. American Association of Clinical Endocrinologists and American College of Endocrinology. 2016 Outpatient glucose monitoring consensus statement. Endocr Pract 2016; 22:231-261.

8. Sherr J,Tauschmann M, BattelinoT, Bock M, Forlenza G, Roman R, Hood K, Maahs D. ISPAD Clinical Practice Consensus Guidelines 2018: Diabetes Technologies. Pediatric Diabetes 2018; 19 (Suppl.27):302-325.

9. American Diabetes Association. Diabetes Technology: Standars of medical care in diabetes-2019. Diabetes Care 2019; 42(Suppl. 1):S71-80.

10. Laffel L, Aleppo G, Buckingham G, Forlenza G, Rasbach L,Tsalikian $E$, et al. A practical approach to using trend arrows on the Dexcom G5 CGM system to manage children and adolescents with diabetes. J Endocr Soc 2017 Dec; 1(12):1461-1476.

11. Murphy HR, Rayman G, Duffield K, et al. Changes in the glycemic profiles of women with type 1 and type 2 diabetes during pregnancy. Diabetes Care 2007 Nov; 30(11):2785-2791.

12. Kröger J, Fasching $P$, Hanaire $H$, et al. Three European retrospective real-world chart review studies to determine the effectiveness of flash glucose monitoring on $\mathrm{HbA} 1 \mathrm{c}$ in adults with type 2 diabetes. Diabetes Ther 2020 Jan;11(1):279-291.

13. Evans $M$, Welsh Z, Ells $S$, et al. The impact of flash glucose monitoring on glycaemic control as measured by $\mathrm{HbA} 1 \mathrm{c}$ : a meta-analysis of Clinical Trials and Real-World Observational Studies. Diabetes Ther 2020 Jan; 11(1):83-95.

14. Battelino T, Danne T, Bergenstal RM, et al. Clinical targets for continuous glucose monitoring data interpretation: recommendations from the international consensus on time in range. Diabetes Care 2019; 42(8):1593-1603.

15. Bergenstal RM, Ahmann AJ, Bailey T, et al. Recommendations for standardizing glucose reporting and analysis to optimize clinical decision making in diabetes: the ambulatory glucose profile (AGP). Diabetes Technol Ther 2013; 15:198-211. 
16. Xing D, Kollman C, Beck RW, et al.; Juvenile Diabetes Research Foundation Continuous Glucose Monitoring Study Group. Optimal sampling intervals to assess long-term glycemic control using continuous glucose monitoring. Diabetes Technol Ther $2011 ; 13: 351-358$.

17. Riddlesworth TD, Beck RW, Gal RL, et al. Optimal sampling duration for continuous glucose monitoring to determine longterm glycemic control. Diabetes Technol Ther 2018; 20:314-316.

18. Beck RW, Connor CG, Mullen DM, Wesley DM, Bergenstal $\mathrm{RM}$. The fallacy of average: how using $\mathrm{HbA} 1 \mathrm{c}$ al one to assess glycemic control can be misleading. Diabetes Care 2017; 40:994-999.

19. Bergenstal RM, Beck RW, Close $K L$, et al. Glucose management indicator (GMI): a new term for estimating A1C from continuous glucose monitoring. Diabetes Care 2018; 41:2275-2280.

20. American Diabetes Association. Diabetes technology: Standards of medical care in diabetes-2019. Diabetes Care 2019; 43(suppl 1):S1-S212.

21. Feig DS, Donovan LE, Corcoy R, et al.; CONCEPTT Collaborative Group. Continuous glucose monitoring in pregnant women with type 1 diabetes (CONCEPTT): a multicentre international randomised controlled trial. Lancet 2017; 390:2347-2359.

22. Lu J, Ma X, Zhou J, et al. Association of time in range, as assessed by continuous glucose monitoring, with diabetic retinopathy in type 2 diabetes. Diabetes Care 2018; 41:2370-2376.

23. Beck RW, Bergenstal RM, Riddlesworth TD, et al. Validation of time in range as an outcome measure for diabetes clinical trials. Diabetes Care 2019; 42:400-405.

24. Beck RW, Bergenstal RM, Cheng P, et al. The relationships between time in range, hyperglycemia metrics, and HbA1c. J Diabetes Sci Technol 2019 Jan (Epub ahead of print). DOI:10.1177/1932296818822496

25. Vigersky RA, Mc Mahon C. The relationship of hemoglobin A1C to time-in-range in patients with diabetes. Diabetes TechnolTher 2019; 21:81-85.

26. Rodbard D. Glucose variability: a review of clinical applications and research developments. Diabetes Technol Ther 2018; 20(suppl 2):S25-S215.

27. Monnier L, Colette C, Wojtusciszyn A, Dejager S, Renard E, Molinari $\mathrm{N}$, et al. Toward defining the threshold between low and high glucose variability in diabetes. Diabetes Care 2017 Jul; 40:832-838.
28. Rodbard D. Hypo and hyperglycemia in relation to the mean, standard deviation, coefficient of variation, and nature of the glucose distribution. Diabetes Technol Ther 2012; 14:868-876.

29. Danne T, Nimri R, Battelino T, et al. International consensus on use of continuous glucose monitoring. Diabetes Care 2017; 40:1631-1640.

30. Litwak L, Querxoli I, Musso C, Dain A, Houssay S, Costa Gil JE. Monitoreo continuo de glucosa, utilidad e indicaciones. Medicina (Buenos Aires) 2019; 79:44-52.

31. Ziegler R, von Sengbusch S, Kröger J, Schubert O, Werkmeister $P$, Deiss D, Siegmund T. Therapy adjustments based on trend arrows using continuous glucose monitoring systems. J Diabetes SciTechnol 2019; 1-11.

32. Borot S, Benhamou P, Atlan C, Bismuth E, Bonnemaison E, Catargi $B$, et al. Practical implementation, education and interpretation guidelines for continuous glucose monitoring: A French position statement. Diabetes Metab 2018; 44:61-72.

33. Aleppo G, Laffel L, Ahmann A, Hirsch I, Kruger D, Peters A, et al. Un enfoque práctico para usar las flechas de tendencia en el sistema Dexcom G5 CGM para el manejo de adultos con diabetes. Journal of the Endocrine Society 2017 Dec; 1(12):14451460.

34. Yaron M, Roitman E, Aharon-Hananel G, Landau Z, Ganz T, Yanuv $\mathrm{I}$, et al. Effect of flash glucose monitoring technology on glycemic control and treatment satisfaction in patients with type 2 diabetes. Diabetes Care 2019 Jul; 42(7):1178-1184.

35. HaakT, Hanaire H, Ajjan R, Hermanns N, Riveline JP, Rayman G. Flash glucose-sensing technology as a replacement for blood glucose monitoring for the management of insulin-treated type 2 diabetes: a multicenter, open-label randomized controIled trial. Diabetes Ther 2017;8:55-73.

36. Website plataformas digitales de FreeStyle Libre. Disponible en: www.freestylelibre.com.ar, www.libreview.com

37. Hirsch I, BattelinoT, Peters AL, Chamberlain JJ, Aleppo G, Bergenstal R. Role of continuous glucose monitoring in diabetes treatment. Arlington (VA): American Diabetes Association; 2018 Aug.

38. Ajjan RA, Cummings $M H$, Jennings $P$, Leelarathna L, Rayman G, Wilmot EG. Optimising use of rate-of-change trend arrows for insulin dosing decisions using the FreeStyle Libre flash glucose monitoring system. Diab Vasc Dis Res 2019 Jan; 16(1):3-12.

39. Chico A, Aguilera E, Ampudia-Blasco FJ, Bellido V, CardonaHernández R, Escalada FJ, et al. Clinical approach to flash glucose monitoring: an expert recommendation. J Diabetes Sci Technol 2020 Jan;14(1):155-164. 